\title{
Current challenges in COVID-19 diagnosis: a narrative review and implications for clinical practice
}

\author{
Gabriella Nucera, ${ }^{1}$ Francesco Chirico, ${ }^{2,3}$ Valentina Raffaelli, ${ }^{1}$ Pietro Marino ${ }^{1}$ \\ ${ }^{1}$ Emergency Department, Fatebenefratelli Hospital, IRCCS Fatebenefratelli and Sacco; ${ }^{2}$ Post-graduate School of Occupational \\ Health, Università Cattolica del Sacro Cuore; ${ }^{3}$ Health Service Department, Italian State Police, Ministry of the Interior, Milano, \\ Italy
}

\begin{abstract}
Early diagnosis of coronavirus disease 2019 (COVID-19) is crucial to early treatment and quarantine measures. In this narrative review, diagnostic tools for COVID-19 diagnosis and their main critical issues were reviewed. The COVID-19 real-time reverse transcriptase-polymerase chain reaction (RT-PCR) test is considered the gold standard test for the qualitative and quantitative detection of viral nucleic acid. In contrast, tests can be used for epidemiological surveys on specific communities, including occupational cohorts, but not for clinical diagnosis as a substitute for swab tests. Computed tomography (CT) scans can be useful for the clinical diagnosis of COVID-19, especially in symptomatic cases. The imaging features of COVID-19 are diverse and depend on the stage of infection after the onset of symptoms. CT sensitivity seems to be higher in patients with positive RT-PCR. Conventional chest sensitivity shows a lower sensitivity. An important diagnostic screening tool is ultrasounds, whose specificity and sensitivity depend on disease severity, patient weight, and operator skills. Nevertheless, ultrasounds could be useful as a screening tool in combination with clinical features and molecular testing to monitor disease progression. Clinical symptoms and non-specific laboratory findings may be useful if used in combination with RT-PCR test and CT-scanning.
\end{abstract}

\section{Introduction}

Coronavirus disease 2019 (COVID-19) caused by severe acute respiratory syndrome-related coronavirus

Correspondence: Francesco Chirico, Post-graduate School of Occupational Health, Università Cattolica del Sacro Cuore, via Umberto Cagni 21, 20162 Milano, Italy.

E-mail: francesco.chirico@unicatt.it

Key words: COVID-19; computed tomography scan; diagnosis; imaging; real time-polymerase chain reaction; SARSCoV-2; serological test; swab test.

Contributions: GN, FC contributed equally to the paper conceptualization; FC, first draft, GN, revision; VR, PM, literature search; PM, supervision. All the authors approved the final version of the paper. The corresponding author attests that all listed authors meet authorship criteria and that no others have been omitted.

Conflict of interests: the authors declare no potential conflict of interests.

Received for publication: 7 February 2021.

Accepted for publication: 9 February 2021.

This work is licensed under a Creative Commons Attribution NonCommercial 4.0 License (CC BY-NC 4.0).

${ }^{\circ}$ Copyright: the Author(s), 2021

Licensee PAGEPress, Italy

Italian Journal of Medicine 2021; 15:129-134

doi:10.4081/itjm.2021.1474
2 (SARS-CoV-2) is an emergent public health crisis threatening the current world health establishment and, therefore, has been declared a pandemic by the World Health Organization. SARS-CoV-2 belongs to the human coronavirus $(\mathrm{HCoV})$ family that targets the lower part of the respiratory tract and causes severe acute respiratory syndrome (SARS). Currently, there are no treatments for this infection. For this reason, the preventive measures established across various countries, which are social distancing, usage of a mask to prevent the entry of the virus into the respiratory tract, quarantine, and other containment measures, are capable of reducing morbidity and mortality in highly susceptible individuals. ${ }^{1,2}$ SARS-CoV-2 is primarily transmitted by inhalation of droplets and indirectly by contact with infected fomites, and via airborne through inhalation of bioaerosols that remain suspended in the air. ${ }^{3}$ The COVID-19 patient usually presents with fever, cough, sore throat, and breathlessness. Currently, available data indicate that most people with the disease have mild symptoms, while about $20 \%$ present with moderate-to-severe disease. About $5 \%$ of these may progress to pneumonia, acute respiratory distress syndrome, and multi-organ dysfunction. ${ }^{4,5}$

While several drugs have demonstrated in vitro activity against SARS-CoV-2 or potential clinical benefits, and current mass vaccination campaigns have just began, early recognition and appropriate treatment of immunologic complications can decrease the morbidity and mortality in COVID-19 infection. ${ }^{6}$ For this reason, early diagnosis of COVID-19 is crucial to both 
early treatment and quarantine measures. In this narrative review, therefore, we describe the primary diagnostic methods and the current challenges concerning the COVID-19 diagnosis.

\section{Discussion}

The diagnosis of COVID-19 is based on a combination of clinical findings, epidemiological history, and exams findings of the patient, which are: chest Xray and tomography (CT-scan) revealing the characteristic images of ground glass; serological testing; non-specific laboratory findings; and most of all, oropharyngeal swab test aimed to demonstrate SARSCoV-2 RNA in respiratory samples, which is the gold diagnostic standard. ${ }^{7}$

In these months, accelerated development of molecular and serological assays across a plethora of molecular and serological assays has been developed across many platforms. There are two main tests available for COVID-19: direct tests (i.e.. molecular tests) that are designed for diagnosing a current infection, and indirect tests (i.e., serological tests) that are designed to ascertain seroconversion upon a previous $(\mathrm{IgG})$ or an early (IgM) infection. A combination of these tests, including nucleic acid amplification tests, direct viral antigen tests, and the rapidly expanding laboratory-based and point of care serological tests, may inform crucial decisions by healthcare providers and policymakers. ${ }^{8}$

The COVID real-time reverse transcriptase-polymerase chain reaction (RT-PCR) test is considered the gold standard test for the qualitative and quantitative detection of viral nucleic acids. Other relevant laboratory methods include enzyme-linked immunoassays (EIA) for viral antibody and antigen detection and serum viral neutralization (SVN) assays for antibody neutralization determination. The key components of viral diagnostic tests are: i) collection of the appropriate sample (blood, nasal swab, and throat swab); ii) availability of the genetic and proteomic sequences of the novel virus for analysis; and iii) rapid and accurate laboratory testing methods. Several point-of-care molecular devices are currently being integrated for fast and accurate diagnosis of SARS-CoV-2 infections. However, diagnostic tests capable of measuring low viral loads for early detection, with low or no crossreactivity with other viral strains, are to be developed. ${ }^{9}$ In the event of suspected symptoms, the molecular swabs should be immediately performed to confirm the diagnosis, isolate the positive subject, and trace his/her contacts. ${ }^{10,11}$ RT-PCR is the frontline diagnostic test for COVID-19 capable of analyzing thousands of specimens in a single day and showing a testing sensitivity of $95 \% .{ }^{9}$ It can be carried out on different types of samples (i.e., nasopharyngeal swab, oropharyngeal swab, bronchoalveolar lavage, tracheal aspirates, and saliva). At present, the US Centers for Disease Control and Prevention (CDC) recommend collecting and testing a nasopharyngeal specimen as the preferred choice for swab-based SARS-CoV-2 testing. For initial diagnostic testing for SARS-CoV-2, the CDC recommends collecting and testing an upper respiratory specimen. Swabs should be placed immediately into a sterile transport tube containing 2-3 mL of either viral transport medium (VTM), amies transport medium, phosphate-buffered saline, or sterile saline unless using a test designed to analyze a specimen directly (i.e., without placement in VTM), such as some point-of-care tests. Testing lower respiratory tract specimens is also an option. For patients who develop a productive cough, sputum can be collected and tested when available for SARS-CoV-2. However, the induction of sputum is not recommended. Under certain clinical circumstances (e.g., those receiving invasive mechanical ventilation), a lower respiratory tract aspirate or bronchoalveolar lavage specimen should be collected and tested as a lower respiratory tract specimen. ${ }^{12}$

Therefore, for individuals having invasive procedures, lower respiratory tract specimens are recommended, if available. ${ }^{8}$ The RT-PCR technique shows some analytical problems such as inadequate procedures for collection, handling, transport and storage of the swabs, manual errors, testing outside the diagnostic window, active viral recombination, and inadequately validated assays, that contribute to jeopardize the diagnostic accuracy. ${ }^{13}$ In literature, authors reported case reports of COVID-19 patients showing a chest computed tomography (CT) picture of pneumonia and multiple negative molecular swabs. ${ }^{14} \mathrm{Cao}$ et al. found some symptomatic COVID-19 patients with positive IgM antibodies and negative nasopharyngeal swab testing. ${ }^{14}$ Other scholars reported symptomatic patients with 2 negative nasopharyngeal swabs and a positive swab on bronchoalveolar lavage sample via bronchoscopy. ${ }^{15,16}$ Ai et al. showed a prevalence of false-negative swabs as $48 \%$ in patients with a high likelihood of being infected and $33 \%$ in probable cases of infections. ${ }^{17}$ All the authors concluded not to rely on swabs without searching for clinical and epidemiological evidence before ruling out a COVID-19 diagnosis. ${ }^{18} \mathrm{~A}$ false negative swabs could be derived by sampling or analytical issues, low viral load, mutations in the viral genome, or active viral recombination. ${ }^{13,15,17}$ The sensitivity of nasopharyngeal test could also depend on the timing with respect to the clinical course of the infection, ${ }^{14,15,17}$ and viral load could depend on the number of days after the onset of the symptoms. In the first 14 days, the diagnostic reliability could be higher in nasal swabs collected by sputum, whereas oropharyngeal swabs would be less 
reliable after 8 days from the onset of symptoms. ${ }^{19,20}$ According to some scholars, the viral loads in throat swabs is most significant at the time of viral onset, ${ }^{21,22}$ and the viral shedding may begin 2-3 days before the appearance of the first symptoms, facilitating presymptomatic or asymptomatic transmission. ${ }^{23}$

Tests for the rapid detection of SARS-CoV-2 antigens have been developed because the rapid diagnosis of COVID-19 patients is essential to reduce the disease spread. However, the sensitivity of these tests is lower than that of RT-PCR, and specificity is awaited, given the potential for cross-reaction with other human $\mathrm{CoV}^{24}$ Therefore, their greatest utility has been suggested for symptomatic patients, when the viral load is at its greatest level. ${ }^{8}$ The target functional receptor of SARS and SARS-CoV-2 viruses is the angiotensin-converting enzyme 2 (ACE2). ${ }^{25}$ Among factors contributing to the false-negative results of naso-pharyngeal and oro-pharyngeal swabs, apart from the sampling technique, the transportation process, and the limited gene(s) detection, Winichakoon suggested the nature of coronavirus itself. ${ }^{15}$ Surface expression of ACE2 was found abundantly on both type I and type II alveolar epithelial cells but minimally on bronchial epithelial cells and negative on the nasal, oral, and nasopharynx samples. ${ }^{15}$ Diagnostic testing suggests that simple throat swabs provide sufficient sensitivity when symptoms are still mild or in the prodromal stage. ${ }^{10}$ Later in the disease, COVID-19 then resembles SARS in terms of replication in the lower respiratory tract, and patients with symptoms of lung affection showed a prolonged viral load in sputum. ${ }^{10}$

COVID-19 diagnosis is based on RT-PCR testing of respiratory samples from nasopharyngeal and oropharyngeal swabs and lower respiratory tract samples whenever possible. Bronchoalveolar lavage and sputum induction should be limited and performed only if indicated and with adequate precautions due to the risk of aerosolization and consequent exposure of healthcare professionals. Tracheal aspirate specimens appear to carry a lower risk of aerosolization and can sometimes be obtained without disconnecting the patient from the ventilator. ${ }^{14}$ Similarly, in a study accounting for CT scan findings among suspected COVID-19 cases, $48 \%$ with negative oropharyngeal or nasal swabs were considered highly likely cases, and $33 \%$ were considered probable cases. ${ }^{17}$

EIA assays are diagnostic methods used to identify antibodies in patients' blood samples or nasopharyngeal swabs. Enzyme-linked immunosorbent assays (ELISAs) for antibody detection against SARS-CoV2 measure the host humoral response. IgM is the first immunoglobulin produced in response to an antigen and is primarily detected during the early onset of disease (3-7 days). IgG is the most abundant im- munoglobulin produced in response to an antigen (725 days) and is maintained in the body after initial exposure and may have a protective role for acquired immunity. ${ }^{9}$ However, the overall sensitivity and specificity indicate the possibility of false negatives and false positives in this testing method. ${ }^{26}$

Since the risk for recurrent infection with SARS$\mathrm{CoV}-2$ is not known for COVID-19, detecting one or two antibodies (IgM and/or IgG) does not necessarily guarantee immunity against reinfection. Furthermore, negative results do not rule out SARS-CoV-2 infection, particularly in those who have been in contact with the virus, and positive results may be due to past or present infection with SARS-CoV. ${ }^{27}$

Several research laboratories have used the EIA platform to develop lateral flow immunoassays (LFIA) for the rapid qualitative detection of SARS$\mathrm{CoV}$. This is designed as a simple, portable diagnostic strip to measure either SARS-CoV-2 antibodies or antigens. The major advantage is that this technique delivers results in $\sim 15 \mathrm{~min}$ and uses visual detection by the naked eye compared to RT-PCR (2-5 days). Finally, the SVN assay is a serological test that measures the ability of a patient's antibodies to neutralize infectivity of SARS-CoV-2 and attenuate infection. However, it is not used for routine diagnosis but is the frontline for this special indication. The specificity and sensitivity of LIFAs are comparable for antibody and antigen assays. ${ }^{9}$

Finally, tests that can be performed at the point-ofcare by less specialized personnel are named point of care tests (POCTs). POCTs are usually rapid tests, and when rapid antigen tests are well-validated, they may be considered for the rapid diagnosis of infected patients. However, these tests tend to have lower sensitivity than RT-PCR, and therefore, even if they may be helpful during an ongoing outbreak when timely access to sensitive molecular testing is unavailable, a negative result should be interpreted by a healthcare professional with caution and based on clinical judgement. ${ }^{28}$

According to a recent meta-analysis on sensitivity and specificity of different serological testing [enzyme-linked immunosorbent assays (ELISAs), lateral flow immunoassays (LFIAs), or chemiluminescent immunoassays (CLIAs)] and immunoglobulin class (IgG, IgM, or both), available evidence does not support the continued use of existing point-of-care serological tests. Indeed, the pooled sensitivity of ELISAs measuring $\operatorname{IgG}$ or IgM was $84.3 \%$ (95\% confidence interval $75.6 \%$ to $90.9 \%$ ), of LFIAs was $66.0 \%$ (49.3\% to $79.3 \%$ ), and of CLIAs was $97.8 \%$ (46.2\% to $100 \%$ ). In all analyses, pooled sensitivity was lower for LFIAs, the potential point-of-care method. ${ }^{29}$ In Italy, the Italian National Institute of Health recommends CLIA and ELISA serological tests with a specificity of at least $95 \%$ and sensitivity of at least $90 \%$. 
However, they can be used for epidemiological surveys on specific communities, including occupational cohorts, but not for clinical diagnosis as a substitute for swab tests. ${ }^{30,31}$

Due to the shortage of kits and false-negative rate of RT-PCR, the Hubei Province, China temporarily used CT scans as a clinical diagnosis for COVID-19. ${ }^{32}$ The imaging features of COVID-19 are diverse and depend on the stage of infection after the onset of symptoms. ${ }^{33}$ The most common hallmark features of COVID-19 include bilateral and peripheral groundglass opacities (areas of hazy opacity) $)^{34}$ and consolidations of the lungs (fluid or solid material in compressible lung tissue). ${ }^{35,36}$ Typical CT manifestations of COVID-19 infection are ground-glass opacities, consolidation, reticular pattern, and crazy paving pattern. Emerging atypical CT manifestations, including airway changes, pleural changes, fibrosis, nodules, etc., were demonstrated in COVID-19 patients. CT manifestations may be associated with the progression and prognosis of COVID-19. ${ }^{37}$ However, CT systems are expensive, require technical expertise, and cannot precisely diagnose COVID-19. ${ }^{33}$ Therefore, even if CT findings are essential for both diagnosis and follow-up, they can be useful, especially for early diagnosis. ${ }^{16,38-40}$ CT sensitivity seems to be higher in patients with positive RT-PCR (86-97\% in different case studies), ${ }^{41}$ and lower in patients with only constitutional and nonrespiratory symptoms. ${ }^{42}$

Although multiple studies suggest CT should be a primary diagnostic tool for coronavirus disease (COVID-19) because they reported sensitivities with CT far superior to that of reverse transcriptase-polymerase chain reaction (RT-PCR) testing, CT has limited sensitivity for COVID-19 and lower specificity than RT-PCR testing. It carries a risk of exposing providers to severe acute respiratory syndrome coronavirus 2 (SARS-CoV-2). Therefore, chest CT should be considered a supplemental diagnostic tool, particularly for patients who show symptoms. ${ }^{43}$

Conventional chest X-ray sensitivity is at around $59 \%{ }^{44}$ A meta-analysis on chest X-ray examinations revealed that most novel coronavirus pneumonia patients presented with bilateral lung injury $(72.9 \%)$, which was primarily characterized by ground-glass opacities $(68.5 \%))^{45}$

Ultrasounds are a crucial diagnostic screening tool, whose sensitivity is estimated to be around $75 \%$; however, they show very low specificity and variability depending on factors such as disease severity, patient weight, and operator skills. Nevertheless, ultrasounds could be helpful as a screening tool in combination with clinical findings and molecular testing. Furthermore, it has been suggested their use for monitoring the progression of the disease, ${ }^{46}$ and distinguish COVID-19 by community-acquired pneumonia
(CAP) ${ }^{47}$ At present, the diagnosis of COVID-19-related pneumonia depends on a combination of laboratory testing and imaging analyses of variable diagnostic efficacy. High-resolution Computed Tomography has been associated with a higher diagnostic accuracy rate than a real-time quantitative polymerase chain reaction-based approach $(\mathrm{P}=0.0041)$, and chest radiography $(\mathrm{P}=0.0100) .^{48}$ Typical CT findings in individuals with COVID-19 were ground-glass opacities, particularly on the peripheral and lower lobes, and bilateral multiple lobular and subsegmental areas of consolidation, especially in ICU patients. ${ }^{49}$ Therefore, it is essential that clinicians utilize a combination of laboratory and radiological testing when possible, to ensure that SARS-CoV-2 is reliably and quickly detected. This allows early isolation and treatment of the infected. ${ }^{48}$

The typical clinical features of COVID-19 are fever, fatigue, and dry cough. Atypical clinical symptoms include expectoration, headache, hemoptysis, nausea, vomiting, and diarrhea. Chemosensory dysfunction, such as loss of smell and taste, is also closely associated with COVID-19 infection but is usually recovered within 2 to 4 weeks after infection. ${ }^{50,51}$

Finally, non-specific laboratory findings have also been described. They include leukopenia and lymphopenia in $80 \%$ of the cases, depletion of CD4 and CD8 lymphocytes, in addition to mild thrombocytopenia, increased inflammatory markers, such as lactate dehydrogenase (LDH), erythrocyte sedimentation rate (ESR), C-reactive protein (CRP), aspartate aminotransferase (AST), troponin, ferritin, creatine kinase (CK) and D-dimer, in addition to the extended prothrombin time. Some studies suggest that changes in the neutrophil/lymphocyte ratio in the severe disease progression of COVID-19 patients are also suggested. ${ }^{7}$ A meta-analysis of some research studies was conducted, and the following abnormalities in blood indicators were found: decreased albumin (75.8\%), increased C-reactive protein (58.3\%), increased lactate dehydrogenase (LDH) (57.0\%), decreased lymphocytes $(43.1 \%)$, and increased erythrocyte sedimentation rate (ESR) $(41.8 \%){ }^{45}$

\section{Conclusions}

In accordance with the literature, although RTPCR has been described as the gold standard for diagnosing COVID-19, several difficulties involve its use. Therefore, a positive test is highly suggestive of true COVID-19, but a negative test does not rule out the disease. ${ }^{7}$ In this case, highly suspected patients and providers in epidemic areas should assume they have the disease and undergo early treatment. In conclusion, only a combination of clinical features, epidemi- 
ological data, laboratory exams, and imaging findings may drive the physician to an appropriate diagnostic hypothesis of COVID-19 infection.

\section{References}

1. Berekaa MM. Insights into the COVID-19 pandemic: Origin, pathogenesis, diagnosis, and therapeutic interventions. Front Biosci (Elite Ed) 2021;13:117-39.

2. Soy M, Keser G, Atagündüz P, et al. Cytokine storm in COVID-19: pathogenesis and overview of anti-inflammatory agents used in treatment. Clin Rheumatol 2020; 39:2085-94.

3. Magnavita N, Sacco A, Chirico F. Covid-19 pandemic in Italy: Pros and cons. Zdrowie Publiczne i Zarządzanie 2020;16:32-5.

4. Chirico F, Sacco A, Bragazzi NL, Magnavita N. Can airconditioning systems contribute to the spread of SARS/MERS/COVID-19 infection? Insights from a rapid review of the literature. Int J Environ Res Public Health 2020;17:6052.

5. Kaswa R, Govender I. Novel coronavirus pandemic: A clinical overview. S Afr Fam Pract (2004) 2020;62:e1-5.

6. Chirico F, Nucera G, Magnavita N. Estimating case fatality ratio during COVID-19 epidemics: Pitfalls and alternatives. J Infect Dev Ctries 2020;14:438-9.

7. Oliveira BA, Oliveira LC, Sabino EC, Okay TS. SARSCoV-2 and the COVID-19 disease: a mini review on diagnostic methods. Rev Inst Med Trop Sao Paulo 2020; 62:e44.

8. La Marca A, Capuzzo M, Paglia T, et al. Testing for SARS-CoV-2 (COVID-19): a systematic review and clinical guide to molecular and serological in-vitro diagnostic assays. Reprod Biomed Online 2020;41:483-99.

9. D'Cruz RJ, Currier AW, Sampson VB. Laboratory testing methods for novel severe acute respiratory syndrome-coronavirus-2 (SARS-CoV-2). Front Cell Dev Biol 2020;8:468.

10. Woelfel R, Corman VM, Guggemos W, et al. Clinical presentation and virological assessment of hospitalized cases of coronavirus disease 2019 in a travel-associated transmission cluster. medRxiv 2020 Mar 8 [Epub ahead of print].

11. Alhazzani W, Møller MH, Arabi YM, et al. Surviving sepsis campaign: guidelines on the management of critically ill adults with coronavirus disease 2019 (COVID-19). Intensive Care Med 2020 Mar 28 [Epub ahead of print].

12. CDC. Interim guidelines for collecting, handling, and testing clinical specimens for COVID-19; Updated 06 Jan 2021. Available from: https://www.cdc.gov/coronavirus/2019-ncov/lab/guidelines-clinicalspecimens.html Accessed: 6 February 2021.

13. Lippi G, Simundic AM, Plebani M. Potential preanalytical and analytical vulnerabilities in the laboratory diagnosis of coronavirus disease 2019 (COVID-19). Clin Chem Lab Med 2020;58:1070-6.

14. Xie X, Zhong Z, Zhao W, et al. Chest CT for typical 2019-nCoV pneumonia: relationship to negative RTPCR testing. Radiology 2020;200343.

15. Winichakoon P, Chaiwarith R, Liwsrisakun C, et al.
Negative nasopharyngeal and oropharyngeal swab does not rule out COVID 19. J Clin Microbiol 2020 [Epub ahead of print].

16. Kumar DS, O'Neill SB, Johnston JC, et al. SARS-CoV-2 infection in a 76-year-old man with negative results for nasopharyngeal swabs and possible nosocomial transmission. CMAJ 2020; cmaj.200641. [Epub ahead of print].

17. Ai T, Yang Z, Hou H, et al. Correlation of chest CT and RT-PCR testing in coronavirus disease 2019 (COVID19) in China: a report of 1014 Cases. Radiology 2020;2020:200642.

18. Chirico F, Nucera G, Magnavita N. Hospital infection and COVID-19: Do not put all your eggs on the "swab" tests [published online ahead of print, 2020 May 27]. Infect Control Hosp Epidemiol 2020;1-2.

19. Pan Y, Zhang D, Yang P, et al. Viral load of SARS-CoV2 in clinical samples. Lancet Infect Dis 2020;20:411.

20. Yang Y, Yang M, Shen C, et al. Laboratory diagnosis and monitoring the viral shedding of 2019-nCoV Infections. medRxiv 2020 [Epub ahead of print].

21. To KK, Tsang OT, Leung WS, et al. Temporal profiles of viral load in posterior oropharyngeal saliva samples and serum antibody responses during infection by SARS-CoV-2: an observational cohort study. Lancet Infect Dis 2020;20:565-74.

22. Zou L, Ruan F, Huang M, et al. SARS-CoV-2 viral load in upper respiratory specimens of infected patients. $\mathrm{N}$ Engl J Med 2020;382:1177-9.

23. He X, Lau EHY, Wu P, et al. Temporal dynamics in viral shedding and transmissibility of COVID-19. Nat Med 2020;26:672-5.

24. Mak GC, Cheng PK, Lau SS, et al. Evaluation of rapid antigen test for detection of SARS-CoV-2 virus. J Clin Virol 2020;129:104500.

25. Li W, Moore MJ, Vasilieva N, et al. Angiotensin-converting enzyme 2 is a functional receptor for the SARS coronavirus. Nature 2003;426:450-4.

26. Corman VM, Landt O, Kaiser M, et al. Detection of 2019 novel coronavirus (2019-nCoV) by real-time RTPCR. Euro Surveill 2020;25:2000045.

27. Guo L, Ren L, Yang S, et al. Profiling early humoral response to diagnose novel coronavirus disease (COVID19). Clin Infect Dis 2020;71:778-85.

28. European Center for Disease Center and Control. Diagnostic testing and screening for SARS-CoV-2. Available from: https://www.ecdc.europa.eu/en/covid-19/latestevidence/diagnostic-testing

29. Lisboa Bastos M, Tavaziva G, Abidi SK, et al. Diagnostic accuracy of serological tests for covid-19: systematic review and meta-analysis. BMJ 2020;370:m2516.

30. Long QX, Liu BZ, Deng HJ, et al. Antibody responses to SARS-CoV-2 in patients with COVID-19. Nat Med 2020 [Epub ahead of print].

31. Ministero della Salute. Direzione Generale della Prevenzione Sanitaria. COVID-19: test di screening e diagnostici. Circolare del 09 Maggio 2020 n. 0016106-09/05/ 2020-DGPRE-DGPRE-P.

32. Wang W, Yan F. Patients with RT-PCR confirmed COVID-19 and normal chest CT. Radiology 2020: 200702.

33. Udugama B, Kadhiresan P, Kozlowski HN, et al. Diagnosing COVID-19: the disease and tools for detection. ACS Nano 2020;14:3822-35. 
34. Kobayashi Y, Mitsudomi T. Management of groundglass opacities: should all pulmonary lesions with ground-glass opacity be surgically resected? Transl Lung Cancer Res 2013;2:354-63.

35. Bernheim A, Mei X, Huang M, et al. Chest CT findings in coronavirus disease-19 (COVID-19): relationship to duration of infection. Radiology 2020 [Epub ahead of print].

36. Pan F, Ye T, Sun P, et al. Time course of lung changes on chest CT during recovery from 2019 novel coronavirus (COVID-19) pneumonia. Radiology 2020 [Epub ahead of print].

37. Ye Z, Zhang Y, Wang Y, et al. Chest CT manifestations of new coronavirus disease 2019 (COVID-19): a pictorial review. Eur Radiol 2020;30:4381-9.

38. Yang W, Yan F. Patients with RT-PCR confirmed COVID-19 and normal chest CT. Radiology 2020 [Epub ahead of print].

39. Yam WC, Chan KH, Poon LL, et al. Evaluation of reverse transcription-PCR assays for rapid diagnosis of severe acute respiratory syndrome associated with a novel coronavirus. J Clin Microbiol 2003;41:4521-4.

40. Feng H, Liu Y, Lv M, et al. A case report of COVID-19 with false negative RT-PCR test: necessity of chest CT. Jpn J Radiol 2020. [Epub ahead of print].

41. Zhuang GH, Shen MW, Zeng LX, et al. Potential falsepositive rate among the 'asymptomatic infected individuals' in close contacts of COVID-19 patients. Zhonghua Liu Xing Bing Xue Za Zhi 2020;41:485-8.

42. Kanne JP, Little BP, Chung JH, et al. Essentials for radiologists on COVID-19: an update-radiology scientific expert panel. Radiology 2020;200527.

43. Waller JV, Kaur P, Tucker A, et al. Diagnostic tools for coronavirus disease (COVID-19): comparing CT and
RT-PCR viral nucleic acid testing. AJR Am J Roentgenol 2020;215:834-8.

44. Bernheim A, Mei X, Huang M, et al. Chest CT findings in coronavirus disease-19 (COVID- 19): relationship to duration of infection. Radiology 2020:200463.

45. Rodriguez-Morales AJ, Cardona-Ospina JA, GutiérrezOcampo E, et al. Clinical, laboratory and imaging features of COVID-19: a systematic review and meta-analysis. Travel Med Infect Dis 2020;34:101623.

46. Pascarella G, Strumia A, Piliego C, et al. COVID-19 diagnosis and management: a comprehensive review. J Intern Med 2020;288:192-206.

47. Tan G, Lian X, Zhu Z, et al. Use of lung ultrasound to differentiate coronavirus disease 2019 (COVID-19) pneumonia from community-acquired pneumonia. U1trasound Med Biol 2020;46:2651-8.

48. Zheng Z, Yao Z, Wu K, Zheng J. The diagnosis of SARS-CoV2 pneumonia: A review of laboratory and radiological testing results. J Med Virol 2020 [Epub ahead of print].

49. Adhikari SP, Meng S, Wu YJ, et al Epidemiology, causes, clinical manifestation and diagnosis, prevention and control of coronavirus disease (COVID-19) during the early outbreak period: a scoping review. Infect Dis Poverty 2020;9:29.

50. Guo G, Ye L, Pan K, et al. New insights of emerging SARS-CoV-2: epidemiology, etiology, clinical features, clinical treatment, and prevention. Front Cell Dev Biol 2020;8:410.

51. Yan CH, Faraji F, Prajapati DP, et al. Association of chemosensory dysfunction and COVID-19 in patients presenting with influenza-like symptoms. Int Forum Allergy Rhinol 2020;10:806-13. 